\title{
Continuity of the Restriction Maps on Smirnov Classes
}

\author{
Yüksel Soykan \\ Department of Mathematics, Art and Science Faculty, Bülent Ecevit University, 67100 Zonguldak, Turkey
}

Correspondence should be addressed to Yüksel Soykan; yuksel_soykan@hotmail.com

Received 2 June 2014; Accepted 19 August 2014; Published 11 September 2014

Academic Editor: Dashan Fan

Copyright (C) 2014 Yüksel Soykan. This is an open access article distributed under the Creative Commons Attribution License, which permits unrestricted use, distribution, and reproduction in any medium, provided the original work is properly cited.

We prove the restriction maps define continuous linear operators on the Smirnov classes for some certain domain with analytic boundary.

\section{Introduction}

As usual, we define the Hardy space $H^{2}=H^{2}(\Delta)$ as the space of all functions $f: z \rightarrow \sum_{n=0}^{\infty} a_{n} z^{n}$ for which the norm $\left(\|f\|=\sum_{n=0}^{\infty}\left|a_{n}\right|^{2}\right)^{1 / 2}$ is finite. Here, $\Delta$ is the open unit disc. For a more general simply connected domain $D$ in the sphere or extended plane $\overline{\mathbb{C}}=\mathbb{C} \cup(\infty)$ with at least two boundary points, and a conformal mapping $\varphi$ from $D$ onto $\Delta$ (i.e., a Riemann mapping function, abbreviation is RMF), a function $g$ analytic in $D$ is said to belong to the Smirnov class $E^{2}(D)$ if and only if $g=(f \circ \varphi) \varphi^{1 / 2}$ for some $f \in H^{2}(\Delta)$ where $\varphi^{\prime 1 / 2}$ is an analytic branch of the square root of $\varphi^{\prime}$. The reader is referred to [1-7] and references therein for the basic properties of these spaces.

Let $C=\left(C_{1}, C_{2}, C_{3}, \ldots, C_{N}\right)$ be an $N$-tuple of closed distinct curves on the sphere $\overline{\mathbb{C}}$ and suppose that, for each $i, 1 \leq i \leq N, C_{i}$ is a circle, a line $\cup\{\infty\}$, an ellipse, a parabola $\cup\{\infty\}$, or a branch of a hyperbola $\cup\{\infty\}$. Let $D_{i}$ be the complementary domain of $C_{i}$. Recall that a complementary domain of a closed $F \subseteq \overline{\mathbb{C}}$ is a maximal connected subset of $\overline{\mathbb{C}}-F$, which must be a domain. For $1 \leq i \leq N$, suppose that $\varphi_{i}: D_{i} \rightarrow \Delta$ is a conformal equivalence (i.e., RMF) and let $\psi_{i}: \Delta \rightarrow D_{i}$ be its inverse. For $1 \leq i \leq N$, let us keep the notations of $C_{i}, D_{i}, \varphi_{i}, \psi_{i}$ fixed until the end of the paper.

In this paper we prove the following.

Theorem 1. Let $1 \leq i, j \leq N$. Suppose that $\Gamma$ is an open subarc of $C_{j}$ and suppose also that $\Gamma \subseteq D_{i}$ if $i \neq j$. Then the restriction $\left.f \rightarrow f\right|_{\Gamma}$ defines a continuous linear operator mapping $E^{2}\left(D_{i}\right)$ into $L^{2}(\Gamma)$.
For similar work regarding restriction maps, see $[8,9]$. Our conjecture is that Theorem 1 is valid if, for each $j, 1 \leq$ $j \leq N, C_{j}$ is a $\sigma$-rectifiable analytic Jordan curve.

There are some similar results for rectifiable curves in Havin's paper [10]. Also the Cauchy projection operator from $L^{p}$ to $E^{p}$ is bounded on all Carleson regular curves; compare the papers of David, starting with [11].

We need the following Theorem to simplify the proof of Theorem 1 .

Theorem 2 (Theorem 1 in [12]). Let $D$ be a complementary domain of $\cup_{i=1}^{N} C_{i}$ and suppose that $D$ is simply connected so that $D_{i}$ is the complementary domain of $C_{i}$ which contains $D$. Then

(i) $\partial D$ is a $\sigma$-rectifiable closed curve and every $f \in E^{2}(D)$ has a nontangential limit function $\tilde{f} \in L^{2}(\partial D)$;

(ii) (Parseval's identity) the map $f \rightarrow \tilde{f}\left(E^{2}(D) \rightarrow\right.$ $\left.L^{2}(\partial D)\right)$ is an isometric isomorphism onto a closed subspace $E^{2}(\partial D)$ of $L^{2}(\partial D)$, so

$$
\begin{array}{r}
\|f\|_{E^{2}(D)}^{2}=\|\tilde{f}\|_{L^{2}(\partial D)}^{2}=\frac{1}{2 \pi} \int_{\partial D}|\tilde{f}(z)|^{2}|d z|, \\
\left(f \in E^{2}(D)\right) .
\end{array}
$$

If $\Gamma \subseteq C_{i}$ is an open subarc, then

$$
\left\|\left.\tilde{f}\right|_{\Gamma}\right\|_{L^{2}(\Gamma)}^{2} \leq\left\|\left.\tilde{f}\right|_{C_{i}}\right\|_{L^{2}\left(C_{i}\right)}^{2}=\|f\|_{E^{2}\left(D_{i}\right)}^{2},
$$


because Parseval's identity is true for the trivial chain $\left(C_{i}\right)$ of curves. Hence Theorem 1 will be proved if the following theorem can be proved.

Theorem 3. Let $1 \leq i \neq j \leq N$. Suppose that $\Gamma$ is an open subarc of $C_{j}$ and that $\Gamma \subseteq D_{i}$. Then the restriction $f \rightarrow$ $\left.f\right|_{\Gamma}$ defines a continuous linear operator mapping $E^{2}\left(D_{i}\right)$ into $L^{2}(\Gamma)$.

\section{Preliminaries for the Proof of Theorem 3}

Let us keep the notation of Theorem 3 fixed for the rest of the paper and let us also agree to use $l$ for arc-length measure.

An arc or closed curve $\gamma$ is called $\sigma$-rectifiable if and only if it is a countable union of rectifiable arcs in $\mathbb{C}$, together with $(\infty)$ in the case when $\infty \in \gamma$. For instance, a parabola without $\infty$ is $\sigma$-rectifiable arc, and a parabola with $\infty$ is $\sigma$ rectifiable Jordan curve. The following definition will simplify the language.

Definition 4. Let $\gamma \subseteq \mathbb{C}$ be a simple $\sigma$-rectifiable arc contained in a simply connected domain $G \subseteq \overline{\mathbb{C}}$. We say that $\gamma$ has the restriction property in $G$ if and only if the map $g \rightarrow$ $\left.g\right|_{\gamma}$ defines a continuous linear operator mapping $E^{2}(G)$ into $L^{2}(\gamma)$.

Thus, the last sentence of Theorem 3 reads " $\Gamma$ has the restriction property in $D_{i}$ '"

Lemma 5 (Invariance Lemma (Lemma 4 in [9])). Let $G_{1}, G_{2} \subseteq \overline{\mathbb{C}}$ be simply connected domains and suppose that $\gamma_{1} \subseteq G_{1} \cap \mathbb{C}, \gamma_{2} \subseteq G_{2} \cap \mathbb{C}$ are simple $\sigma$-rectifiable arcs. If $\chi: G_{1} \rightarrow G_{2}$ is a conformal equivalence onto $G_{2}$ and $\chi\left(\gamma_{1}\right)=\gamma_{2}$, then $\gamma_{1}$ has the restriction property in $G_{1}$ if and only if $\gamma_{2}$ has the restriction property in $G_{2}$.

Corollary 6. Theorem 3 is true; that is, $\Gamma$ has the restriction property in $D_{i}$, if and only if $\varphi_{i}(\Gamma)$ has the restriction property in $\Delta$, for some $R M F \varphi_{i}: D_{i} \rightarrow \Delta$.

A subarc $\gamma$ of $\Gamma$ has the restriction property in $D_{i}$ if and only if $\varphi_{i}(\gamma)$ has the restriction property in $\Delta$. Corollary 6 will be used in the following way. $\Gamma$ will be written as the union of finitely many subarcs and we will show that each of these subarcs has the restriction property in $D_{i}$; it will then follow that $\Gamma$ itself has the required restriction property. Three different kinds of subarc will be considered.

Definition 7. A subarc $\gamma \subseteq \Gamma$ is said to be of type I if and only if $\bar{\gamma} \subseteq D_{i}$ (i.e., both of its end-points $a, b$ belong to $D_{i}$ ).

Lemma 8 (Lemma 6 in [9]). Let $\gamma$ be a subarc of $\Gamma$ and suppose that $\varphi_{i}, \theta_{i}$ are Riemann mapping functions for $D_{i}$.

(i) $\varphi_{i}(\gamma)$ has the restriction property in $\Delta$ if and only if $\theta_{i}(\gamma)$ has the restriction property in $\Delta$;

(ii) $\varphi_{i}(\gamma)$ is rectifiable if and only if $\theta_{i}(\gamma)$ is rectifiable;

(iii) if $\gamma$ is of type I, then $\overline{\varphi_{i}(\gamma)} \subseteq \Delta$ and $\varphi_{i}(\gamma)$ is rectifiable;

(iv) if $\gamma$ is of type I, it has the restriction property in $D_{i}$.

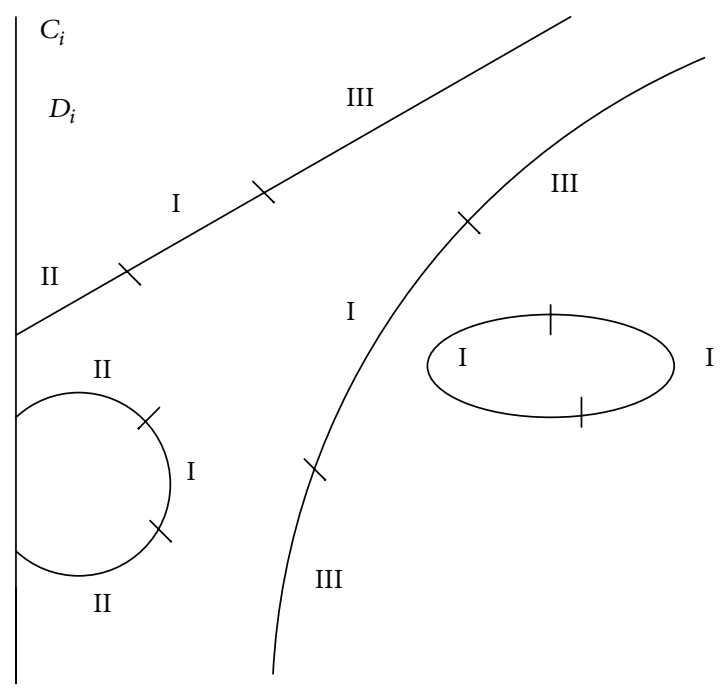

Figure 1: Type I, II, and III arcs.

We can now "ignore" subarcs of $\Gamma$ whose closure (in $\overline{\mathbb{C}}$ ) is contained in $D_{i}$. We will now restrict our attention to subarcs of $\Gamma$ with a single end-point $a \in \partial D_{i}$, the other being in $D_{i}$. There are two types, depending on whether $a \in \mathbb{C}$ or $a=\infty$.

Definition 9. (i) An open subarc $\gamma$ of $\Gamma$ is of type II if and only if it has an end-point $a \in \partial D_{i} \cap \mathbb{C}$ and $\bar{\gamma}-(a) \subseteq D_{i} \cap \mathbb{C}$.

(ii) In the case where $C_{i}$ is unbounded (so that $\infty \in \partial D_{i}$ ) an open subarc $\gamma \subseteq \Gamma$ is of type III if and only if $\infty$ is an endpoint of $\gamma$ and $\bar{\gamma}-(\infty) \subseteq D_{i}$.

Modulo a finite subset of $D_{i}, \Gamma$ is the union of at most three open subarcs, each of which is of type I, II, or III; see Figure 1.

If $\gamma$ is a type II or type III subarc of $\Gamma$ then $\varphi_{i}(\gamma)$ is a simple open analytic arc in $\Delta$ with one end-point on the circle $\mathbb{T}$ and the other in $\Delta$. We will show that $\varphi_{i}(\gamma)$ has the restriction property in $\Delta$ using the powerful Carleson theorem (Theorem 11 below).

Definition 10 (see [1, p.157]). For $0<h<1$ and $0 \leq \theta<2 \pi$, let $C_{\theta h}=\{z \in \mathbb{C}: 1-h \leq|z| \leq 1, \theta \leq \arg z \leq \theta+h\}$. A positive regular Borel measure $\mu$ on $\Delta$ is called a Carleson measure if there exists a positive constant $M$ such that $\mu\left(C_{\theta h}\right) \leq M h$, for every $h$ and every $\theta$.

Theorem 11 (see [1, p. 157, Theorem 9.3] or see [13, p. 37]). Let $\mu$ be a finite positive regular Borel measure on $\Delta$. In order that there exists a constant $C>0$ such that

$$
\int_{\Delta}|f(z)|^{2} d \mu(z) \leq C\|f\|^{2}, \quad \forall f \in H^{2}(\Delta),
$$

it is necessary and sufficient that $\mu$ be a Carleson measure.

To complete the proof of Theorem 3 it is sufficient to show that arc-length measure on $\varphi_{i}(\gamma)$ is a Carleson measure whenever $\gamma$ is of type II or III.

It will be useful to use arc-length to parametrize $\gamma$ and $\varphi_{i}(\gamma)$. Recall that a compact $\operatorname{arc} \sigma$ is called smooth if there exists some parametrization $g:[a, b] \rightarrow \sigma$ such that $g \in$ 
$C^{1}[a, b]$ and $g^{\prime}(t) \neq 0, \forall t \in[a, b]$. Note that if $\sigma$ is smooth, then it is rectifiable; that is,

$$
l(\sigma)=\int_{a}^{b}\left|g^{\prime}(t)\right| d t<\infty
$$

To define the arc-length parametrization of $\sigma$ put $s=$ $s(t)=\int_{a}^{t}\left|g^{\prime}(u)\right| d u$ for $a \leq t \leq b$ so that $0 \leq s \leq \ell(\sigma)$. Then $s^{\prime}(t)=\left|g^{\prime}(t)\right|$ and $t \rightarrow s(t)([a, b] \rightarrow[0, \ell])$ is $C^{1}$ with strictly positive derivative. Hence also its inverse $s \rightarrow$ $t(s)([0, \ell] \rightarrow[a, b])$ is $C^{1}$ with strictly positive derivative. Recall that the arc-length parametrization of the smooth arc $\sigma$ is the map $h:[0, \ell] \rightarrow \sigma$ satisfying $h(s)=$ the point on $\sigma$ length $s$ from the initial point $(g(a))\}$; that is, $h(s)=$ $g(t(s)) 0 \leq s \leq \ell$.

Since $h^{\prime}(s)=g^{\prime}(t(s)) t^{\prime}(s), h \in C^{1}[0, \ell]$, with nonzero derivative, necessarily $\left|h^{\prime}(s)\right|=1$ since

$$
h^{\prime}(s(t))=g^{\prime}(t) t^{\prime}(s)=\frac{g^{\prime}(t)}{s^{\prime}(t)}=\frac{g^{\prime}(t)}{\left|g^{\prime}(t)\right|} .
$$

We need the following lemma.

Lemma 12 (Theorem 1 in [14]). Let $\sigma \subseteq \bar{\Delta}$ be a smooth simple arc with arc-length parametrization $g \in C^{1}[0, \ell]$. Suppose that $|g(0)|=1,|g(s)|<1$ for $0<s \leq \ell$. Then arc-length measure on $\sigma \cap \Delta$ is a Carleson measure; hence $\sigma \cap \Delta$ has the restriction property in $\Delta$.

\section{Type II Subarcs}

The following lemma gives the continuity of the restriction map for finite end-points.

Lemma 13. A type II arc $\gamma \subseteq \Gamma \subseteq D_{i}$ has the restriction property in $D_{i}$.

Proof. By Lemmas 12 and 5 it is sufficient to show that $\overline{\varphi_{i}(\gamma)}$ is a smooth arc in $\bar{\Delta}$. Suppose that $\gamma$ has end-points $a \in \partial D_{i} \cap$ $\mathbb{C}$ and $b \in D_{i} \cap \mathbb{C}$, so that $\bar{\gamma}=\gamma \cup(a) \cup(b)$. Clearly $\bar{\gamma}$ is a smooth arc. Because $C_{i}$ is an open analytic arc, $\varphi_{i}$ can be continued analytically into a neighbourhood $U$ of $a$ so as to be conformal in $D_{i} \cup U$. This means that $\varphi_{i}$ is conformal in a neighbourhood of $\bar{\gamma}$ and so $\overline{\varphi_{i}(\gamma)}=\varphi_{i}(\bar{\gamma})$ is a smooth arc in $\bar{\Delta}$ with $\left|\varphi_{i}(a)\right|=1$ and $\varphi_{i}(\bar{\gamma}-(a)) \subseteq \Delta$. The result now follows from Lemmas 12 and 5.

We have now made a good deal of progress because of the following.

Lemma 14. Theorem 3 is true if $C_{i}$ is a circle or an ellipse.

Proof. In this case $\Gamma$ is a finite union of type I and type II arcs only, so the result follows by Lemma 8(iv) and Lemma 13.

\section{Type III Subarcs}

The proof of Theorem 3 will be completed by showing that every type III arc in $D_{i}$ has the restriction property in $D_{i}$. We have an open subarc $\gamma$ of an open subarc $\Gamma$ of $C_{j}$ and $\Gamma \subseteq D_{i}$. In this case $\infty$ is an end-point of $\gamma$ and $\infty \in \partial D_{i}$, so both $C_{i}$ and $C_{j}$ are unbounded. We will use the same strategy we used for type II arcs in Lemma 13; we show that $\sigma=\overline{\varphi_{i}(\gamma)}$ is a smooth arc in $\Delta$ as in Lemma 12 , so that $\varphi_{i}(\gamma)$ has the restriction property in $\Delta$ and so $\gamma$ has the restriction property in $D_{i}$. The proof is more complicated because conformality of $\varphi_{i}$ at $\infty$ cannot necessarily be used. Instead we make use of the fact that as $z \rightarrow \infty$ along $\gamma$, the unit tangent vector of $\gamma$ at $z$ tends to a limit. The following two Lemmas help us exploit this fact.

Lemma 15. Let $g \in C^{1}[0, \infty)$ with $g^{\prime}(t) \neq 0(t \geq 0)$. Suppose that $c \in \mathbb{C}$ and

$$
\lim _{t \rightarrow \infty} g(t)=c
$$

$$
\lim _{t \rightarrow \infty} \frac{g^{\prime}(t)}{\left|g^{\prime}(t)\right|}=\omega, \quad(|\omega|=1)
$$

exist. Define $\sigma=g([0, \infty)) \cup(c)$. Then

(i) $\sigma$ is a compact arc,

(ii) $\sigma$ is rectifiable,

(iii) $\sigma$ is smooth.

Proof. (i) Define $f$ on $[0,1]$ by

$$
f(t)= \begin{cases}g\left(\tanh ^{-1} t\right) & 0 \leq t<1 \\ c & t=1\end{cases}
$$

Then $f \in C[0,1]$ is a continuous parametrization of $\sigma$.

(ii) To prove that $\sigma$ is rectifiable, it suffices to show that, for some $T>0, \int_{T}^{\infty}\left|g^{\prime}(u)\right| d u<\infty$. Let $\varepsilon(t)=\omega-\left(g^{\prime}(t) /\left|g^{\prime}(t)\right|\right)$. So $\varepsilon(t) \rightarrow 0$ as $t \rightarrow \infty$. Choose $T \geq 0$ such that $|\varepsilon(t)| \leq 1 / 2$ for $t \geq T$. Then, for $t \geq T$,

$$
\left|g^{\prime}(t)\right|(1-\bar{\omega} \varepsilon(t))=\bar{\omega} g^{\prime}(t)
$$

Hence

$$
\begin{gathered}
\int_{T}^{t}\left|g^{\prime}(u)\right|(1-\bar{\omega} \varepsilon(u)) d u=\bar{\omega}(g(t)-g(T)), \quad(t>T), \\
|\varepsilon| \leq \frac{1}{2} \Longrightarrow \operatorname{Re}(1-\bar{\omega} \varepsilon) \geq \frac{1}{2} \Longrightarrow 2 \operatorname{Re}(1-\bar{\omega} \varepsilon) \geq 1
\end{gathered}
$$

So

$$
\begin{aligned}
& \int_{T}^{t}\left|g^{\prime}(u)\right| d u \\
& \quad \leq 2 \int_{T}^{t}\left|g^{\prime}(u)\right| \operatorname{Re}(1-\bar{\omega} \varepsilon(u)) d u \\
& \quad=2 \operatorname{Re}(\bar{\omega}(g(t)-g(T))) \\
& \quad \longrightarrow 2 \operatorname{Re}(\bar{\omega}(c-g(T))) \quad \text { as } t \rightarrow \infty,
\end{aligned}
$$




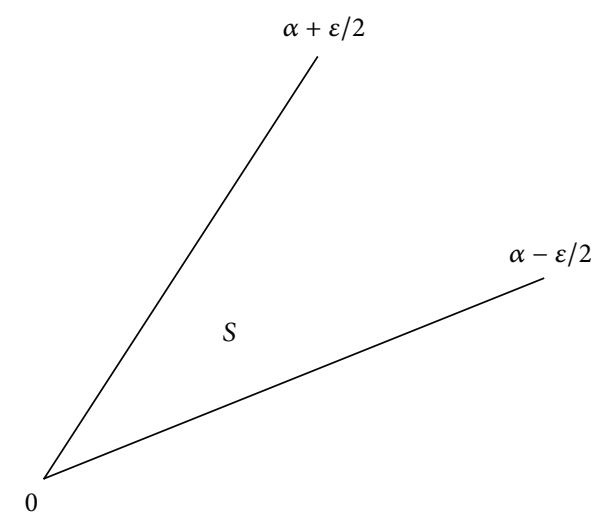

FIgURE 2: The sector $S$.

and hence

$$
\int_{T}^{\infty}\left|g^{\prime}(u)\right| d u<\infty
$$

which establishes the rectifiability of $\sigma$.

(iii) Let $h:[0, \ell] \rightarrow \sigma$ be the arc-length parametrization of $\sigma$. Then $h \in C[0, \ell], h(s)=g(t)$ where $\int_{0}^{t}\left|g^{\prime}(u)\right| d u=s$ and $s^{\prime}(t)=\left|g^{\prime}(t)\right|$. Therefore the map $t \rightarrow s([0, \infty) \rightarrow[0, \ell))$ is $C^{1}$ with strictly positive derivative. So the inverse map $s \rightarrow$ $t([0, \ell) \rightarrow[0, \infty))$ is $C^{1}$. Since $t(s(t)) \equiv t$ and $t^{\prime}(s)=1 / s^{\prime}(t)$ where $0 \leq t \leq \infty$ and $0 \leq s \leq \ell$, it follows that

$\lim _{s \rightarrow \ell} h^{\prime}(s)=\lim _{t \rightarrow \infty} g^{\prime}(t) t^{\prime}(s)=\lim _{t \rightarrow \infty} \frac{g^{\prime}(t)}{s^{\prime}(t)}=\lim _{t \rightarrow \infty} \frac{g^{\prime}(t)}{\left|g^{\prime}(t)\right|}=\omega$.

Hence $h^{\prime}$ is continuous and so $h \in C^{1}[0, \ell]$.

Lemma 16. Let $k \in C^{1}[0, \infty)$ with $k^{\prime}(t) \neq 0(t \geq 0)$ and suppose that $k(t) \rightarrow \infty$ as $t \rightarrow+\infty$. Then, if $|\omega|=1$,

$$
\frac{k^{\prime}(t)}{\left|k^{\prime}(t)\right|} \longrightarrow \omega \Longrightarrow \frac{k(t)}{|k(t)|} \longrightarrow \omega
$$

Proof. Write $\omega=e^{i \alpha}$. Choose $T^{\prime}$ such that $t \geq T^{\prime} \Rightarrow$ $\operatorname{Re} e^{-i \alpha}\left(k^{\prime}(t) /\left|k^{\prime}(t)\right|\right)>0$. Then using $\widehat{\arg }$ to denote the principal value of arg we see that

$$
\theta(t)=\alpha+\widehat{\arg } e^{-i \alpha} \frac{k^{\prime}(t)}{\left|k^{\prime}(t)\right|}
$$

is a branch of $\arg \left(k^{\prime} /\left|k^{\prime}\right|\right)$ and hence also of $\arg k^{\prime}$ on $\left[T^{\prime}, \infty\right)$ which tends to $\alpha$ as $t \rightarrow \infty$. We will find a branch $\vartheta$ of $\arg k$ which also tends to $\alpha$ as $t \rightarrow \infty$.

Let $\varepsilon>0$. Choose $T$ such that $t \geq T \geq T^{\prime} \Rightarrow \alpha-\varepsilon / 2 \leq \theta \leq$ $\alpha+\varepsilon / 2$. Now $k(t)-k(T)=\int_{T}^{t} k^{\prime}(u) d u$ is a limit of Riemann sums $\sum\left(t_{i+1}-t_{i}\right) k^{\prime}\left(\xi_{i}\right)$.

The sector $S$ (see Figure 2) is closed under addition and multiplication by positive scalars; therefore

$$
k(t)-k(T) \in S \quad \text { for } t \geq T .
$$

So there is an argument $\mu(t)$ of $k(t)-k(T)$ satisfying

$$
\alpha-\frac{\varepsilon}{2} \leq \mu(t) \leq \alpha+\frac{\varepsilon}{2} \quad(t \geq T) .
$$

Now $k(t) /(k(t)-k(T)) \rightarrow 1$ as $t \rightarrow \infty$. So

$$
\exists T_{1} \geq T \text { such that } t \geq T_{1} \Longrightarrow-\frac{\varepsilon}{2}<\widehat{\arg } \frac{k(t)}{k(t)-k(T)}<\frac{\varepsilon}{2} .
$$

If we define

$$
\vartheta(t)=\mu(t)+\widehat{\arg } \frac{k(t)}{k(t)-k(T)} \quad\left(t \geq T_{1}\right),
$$

then $\vartheta(t)$ is an argument of $k(t)$ and

$$
t \geq T_{1} \Longrightarrow|\vartheta(t)-\alpha|<\frac{\varepsilon}{2}+\frac{\varepsilon}{2}=\varepsilon
$$

Hence also

$$
\left|\frac{k(t)}{|k(t)|}-\omega\right|=\left|e^{i \vartheta(t)}-e^{i \alpha}\right|<\varepsilon .
$$

Consequently,

$$
\frac{k(t)}{|k(t)|} \longrightarrow \omega=e^{i \alpha}
$$

and our Lemma is proved.

There are now four cases to prove depending on the geometry of $C_{i}$ and $D_{i}$.

4.1. Case 1: $D_{i}$ Is a Half-Plane. The following lemma will be needed here and in Case 2.

Lemma 17. Let $G$ be the open right half-plane $\operatorname{Re} z>0$ and let $\theta(z)=(z-1) /(z+1)$ so that $\theta$ is a Riemann mapping function for $G$. Let $k:[0, \infty) \rightarrow G$ be an injective $C^{1}$ function such that $k^{\prime}(t) \neq 0$, for all $t \geq 0$, and $\lim _{t \rightarrow \infty} k(t)=\infty$. Let $\rho$ be the (simple) arc parametrized by $k$. If $\lim _{t \rightarrow \infty}\left(k^{\prime}(t) /\left|k^{\prime}(t)\right|\right)=$ $\omega$ (with $|\omega|=1$ ), then $\sigma=\overline{\theta(\rho)}$ satisfies the hypothesis of Lemma 12 and, hence, $\rho$ has the restriction property in $G$.

Proof. Put $g=\theta \circ k$, so that $g \in C^{1}[0, \infty)$ parametrizes $\theta(\rho)$. Clearly $g(t) \rightarrow 1$ as $t \rightarrow \infty$. Now $g$ satisfies the hypothesis of Lemma 15, for we can show that $g^{\prime}(t) /\left|g^{\prime}(t)\right| \rightarrow \omega^{-1}$ as $t \rightarrow \infty$. Since $\theta^{\prime}(z)=2 /(z+1)^{2}$ it follows that

$$
\begin{aligned}
\frac{g^{\prime}(t)}{\left|g^{\prime}(t)\right|} & =\frac{|1+k(t)|^{2}}{(1+k(t))^{2}} \frac{k^{\prime}(t)}{\left|k^{\prime}(t)\right|} \\
& =\frac{k^{\prime}(t)}{\left|k^{\prime}(t)\right|} \frac{|k(t)|^{2}}{(k(t))^{2}} \frac{|1+1 / k(t)|^{2}}{(1+1 / k(t))^{2}} \\
& \longrightarrow \omega^{-1},
\end{aligned}
$$

using Lemma 16.

So $\sigma=g[0, \infty) \cup\left(\omega^{-1}\right)$ satisfies Lemma 12 ; hence $g[0, \infty)$ has the restriction property in $\Delta$. But $g[0, \infty)=\theta(\rho)$ and, therefore, by Lemma 5, $\rho$ has the restriction property in $G$. 
Now suppose that $C_{i}$ is a line and $D_{i}$ is a half-plane. By Invariance Lemma 5 with a linear equivalence $\chi(z)=$ $\alpha z+\beta(\alpha \neq 0)$ we can assume that $C_{i}$ is the imaginary axis and that $D_{i}=G$, the open right half-plane, as above. If $\gamma \subseteq D_{i}$ is a type III arc, it is a subarc of a line, parabola, or hyperbola component. Obviously $\gamma$ has a parametrization $k$ as in Lemma 17. Hence $\gamma$ has the restriction property in $D_{i}$.

4.2. Case 2: $D_{i}$ Is the Concave Complementary Domain of a Parabola. Any two parabolas are conformally equivalent via a linear equivalence: $\mu(z)=a z+b(a, b \in \mathbb{C}, a \neq 0)$. So assume that $C_{i}$ is the parabola

$$
y^{2}=4(1-x)
$$

and that $D_{i}$ is the complementary domain to the "right" of $C_{i}$.

The function

$$
w \longrightarrow(1+w)^{2}
$$

maps the open right half-plane $G$ conformally onto $D_{i}$ and the imaginary axis onto $C_{i}$. Its inverse is the function

$$
\vartheta(z)=z^{1 / 2}-1, \quad\left(z \in D_{i}\right),
$$

where $z^{1 / 2}$ is the principal square-root of $z$ (here and throughout all standard multivalued functions will take their principal values).

Now let $\gamma \subseteq D_{i}$ be a type III arc. Because $G$ is conformally equivalent to $D_{i}$ via $\vartheta$ it will be sufficient to show that the arc $\vartheta(\gamma) \subseteq G$ has a parametric function $k$ as in Lemma 17 . Letting $h$ be the arc-length parametrization of $\gamma$, then $h \in$ $C^{1}[0, \infty),\left|h^{\prime}(t)\right| \equiv 1$ and $h(t) \rightarrow \infty$ as $t \rightarrow \infty$, and $h$ is injective.

Now $\gamma$ is a subarc of a line, parabola, or hyperbola component. Hence as $z \rightarrow \infty$ along $\gamma$ the unit tangent vector at $z$ tends to a limit $\omega(|\omega|=1)$. Thus

$$
\lim _{t \rightarrow \infty} \frac{h^{\prime}(t)}{\left|h^{\prime}(t)\right|}=\lim _{t \rightarrow \infty} h^{\prime}(t)=\omega,
$$

and therefore

$$
\lim _{t \rightarrow \infty} \frac{h(t)}{|h(t)|}=\omega
$$

by Lemma 16.

Put $k=\vartheta \circ h$. Then $k$ is an injective parametric function for $\vartheta(\gamma)$. Clearly $k \in C^{1}[0, \infty), k(t) \rightarrow \infty$ as $t \rightarrow \infty$, and

$$
k^{\prime}(t)=\vartheta^{\prime}(h(t)) h^{\prime}(t) \neq 0, \quad \forall t \geq 0 .
$$

Moreover,

$$
\frac{k^{\prime}(t)}{\left|k^{\prime}(t)\right|}=\frac{|h(t)|^{1 / 2}}{h(t)^{1 / 2}} \frac{h^{\prime}(t)}{\left|h^{\prime}(t)\right|} \longrightarrow \omega^{1 / 2} .
$$

So $k$ is as in Lemma 17, which shows that $\gamma$ has the restriction property in $D_{i}$.

Remark 18. The notation $\omega^{1 / 2}$ is ambiguous when $\omega=-1$ ( $\gamma$ could be part of another parabola). But, because type I arcs can be ignored, we can assume that either $\gamma$ is contained entirely in the upper half-plane, in which case $(-1)^{1 / 2}=i$, or else $\gamma$ is in the lower half-plane and $(-1)^{1 / 2}=-i$.
4.3. Case 3: $D_{i}$ Is the Convex Complementary Domain of a Parabola. In this case the parabola

$$
y^{2}=4\left(\frac{\pi}{4}\right)^{2}\left(\left(\frac{\pi}{4}\right)^{2}-x\right)
$$

will be chosen for $C_{i}$, and $D_{i}$ will be the complementary domain to the "left" of $C_{i}$. This choice is made because then we have the relatively simple Riemann mapping function

$$
\varphi_{i}(z)=\tan ^{2}\left(z^{1 / 2}\right), \quad\left(z \in D_{i}\right) .
$$

This function maps the real interval $\left(-\infty,(\pi / 4)^{2}\right)$ in an increasing fashion onto $(-1,1)$, and so it maps the upper/lower half of $D_{i}$ onto the upper/lower half of $\Delta$. The formula for $\varphi_{i}$ is indeterminate on $(-\infty, 0]$, but these singularities are removable and the formula

$$
\varphi_{i}(x)=-\tanh ^{2}(-x)^{1 / 2}
$$

can be used to define $\varphi_{i}(x)$, for negative $x$. This mapping will be examined in detail in a moment, but first we dispose of a trivial case and make some simple observations.

Let $\gamma \subseteq D_{i}$ be a type III arc. If $\gamma$ is a real interval $(-\infty, a)$, with $a<(\pi / 4)^{2}$, then $\varphi_{i}(\gamma)$ is a subinterval of $(-1,1)$ which obviously has the restriction property in $\Delta$. So this case is trivial and needs no more attention.

The following observations are elementary.

(i) If $\gamma$ is part of another line, then it must be parallel to $\mathbb{R}$ and certainly disjoint from $(-\infty, 0]$.

(ii) If $\gamma$ is part of another parabola $C_{j}$, then $C_{j}$ must be symmetric about $\mathbb{R}$ and have an equation of the form

$$
y^{2}=4 a(b-x)
$$

where $0<a \leq(\pi / 4)^{2}, b \leq(\pi / 4)^{2}$.

(iii) If $\gamma$ is part of a hyperbola, then its asymptote must be parallel to $\mathbb{R}$.

(iv) In all (nontrivial) cases $\gamma$ intersects $(-\infty, 0]$ in at most two points. So, because type I arcs can be ignored there is no loss of generality in assuming that $\operatorname{Im} z$ has constant sign on $\gamma$ and that $\operatorname{Re} z<0$ on $\gamma$.

(v) Hence, for definiteness, we can assume that $\gamma$ is contained in the open second quadrant.

(vi) In all cases $y^{2} / x$ tends to a limit as $z \rightarrow \infty$ along $\gamma$. If $\gamma$ is part of a line or hyperbola, the limit is 0 , and if $\gamma$ is part of the parabola in (ii) above the limit is $-4 a$. For future reference let us note that

$$
0 \leq \lim \frac{y^{2}}{4|x|} \leq\left(\frac{\pi}{4}\right)^{2} .
$$

(vii) Because the lim in (34) exists and because type I arcs can be ignored, we can assume that

$$
\frac{y^{2}}{x^{2}}<1, \quad \text { on } \gamma
$$


Now let $\gamma$ be type III arc in $D_{i}$ as in (v) and (vi). We will show that $\varphi_{i}(\gamma)$ has the restriction property in $\Delta$. To elucidate $\varphi_{i}(\gamma)$ it is convenient to work backwards, examining the mapping properties of the square map $\left(z \rightarrow z^{2}\right)$, then tan, and then the principal square root.

Lemma 19. Let $\Delta^{+}$be the open semidisc

$$
\Delta^{+}=\{z \in \mathbb{C}:|z|<1, x>0\} .
$$

If $\sigma^{\prime}$ is a smooth simple arc in $\overline{\Delta^{+}}$, if $i$ is an end-point of $\sigma^{\prime}$, and if $\sigma^{\prime}-\{i\} \subseteq \Delta^{+}$, then the arc

$$
\sigma=\left\{z^{2}: z \in \sigma^{\prime}\right\}
$$

is a smooth simple arc in $\bar{\Delta}$ satisfying the hypothesis of Lemma 12, so that $\sigma-\{-1\}$ has the restriction property in $\Delta$.

Proof. This is clear: the square map $z \rightarrow z^{2}$ is conformal in a neighbourhood of $\sigma^{\prime}$.

Now let $S$ be the open strip

$$
S=\left\{z \in \mathbb{C}: 0<x<\frac{\pi}{4}\right\}
$$

It is well known that tan maps $S$ conformally onto $\Delta^{+}$. The imaginary axis is mapped to the vertical part of $\partial \Delta^{+}$, and the line $\pi / 4+i \mathbb{R}$ is mapped to the semicircular part of $\partial \Delta^{+}$. Moreover, if $z$ tends to infinity in $S$ in such a way that $y \rightarrow$ $+\infty$, then $\tan z \rightarrow i$.

Lemma 20. Let $k \in C^{1}[0, \infty)$ be injective and satisfy $k^{\prime}(t) \neq$ 0 , for $t \geq 0$. Suppose also that

(i) $k(t) \in S$ for all $t \geq 0$,

(ii) $\operatorname{Im} k(t) \rightarrow+\infty$ as $t \rightarrow+\infty$,

(iii) $\lim _{t \rightarrow \infty} \operatorname{Re} k(t)=x_{0}$ exists $\left(0 \leq x_{0} \leq \pi / 4\right)$,

(iv) $\lim _{t \rightarrow \infty}\left(k^{\prime}(t) /\left|k^{\prime}(t)\right|\right)=i$.

If $\gamma^{\prime}$ is the arc parametrized by $k$, then $\sigma^{\prime}=\left(\tan \gamma^{\prime}\right) \cup\{i\}$ satisfies the hypothesis of Lemma 19, so that $\tan ^{2} \gamma^{\prime}$ has the restriction property in $\Delta$.

Proof. Let $g=\tan \circ k$, so that $g$ parametrizes $\gamma^{\prime}$ and $\tan \gamma^{\prime}=$ $g[0, \infty)$. Now $g \in C^{1}[0, \infty), g^{\prime}(t) \neq 0$, for all $t \geq 0$, and $g(t) \rightarrow i$ as $t \rightarrow+\infty$. Lemma 15 will be used to show that $\sigma^{\prime}=g[0, \infty) \cup(i)$ satisfies the hypothesis of Lemma 19. For all $t \geq 0$,

$$
\frac{g^{\prime}(t)}{\left|g^{\prime}(t)\right|}=\frac{|\cos k(t)|^{2}}{(\cos k(t))^{2}} \frac{k^{\prime}(t)}{\left|k^{\prime}(t)\right|}
$$

Let $k(t)=x(t)+i y(t)$. Since $x(t) \rightarrow x_{0}$ and $y(t) \rightarrow+\infty$, as $t \rightarrow+\infty$, and because $\cos x, \cosh y>0$ on $\gamma$,

$$
\begin{aligned}
\frac{|\cos k(t)|^{2}}{\cos ^{2} k(t)} & =\left(\frac{|\cos x(t) \cosh y(t)-i \sin x(t) \sinh y(t)|}{\cos x(t) \cosh y(t)-i \sin x(t) \sinh y(t)}\right)^{2} \\
& =\frac{|1-i \tan x(t) \tanh y(t)|^{2}}{(1-i \tan x(t) \tanh y(t))^{2}} \\
& \longrightarrow \frac{\left|1-i \tan x_{0}\right|^{2}}{\left(1-i \tan x_{0}\right)^{2}} .
\end{aligned}
$$

So $\lim _{t \rightarrow \infty}\left(g^{\prime}(t) /\left|g^{\prime}(t)\right|\right)$ exists.

The function

$$
\vartheta(z)=z^{1 / 2}
$$

maps $D_{i}-(-\infty, 0]$ conformally onto the vertical strip $S$ as above. The limiting values of $\vartheta$ from above and below a point $x$ on $(-\infty, 0]$ are at $\pm i(-x)^{1 / 2}$, respectively. Now $\tan$ maps $S$ conformally onto $\Delta^{+}$and $\tan \pm i(-x)^{1 / 2}=$ $\pm i \tanh (-x)^{1 / 2}$. Finally the square function maps $\Delta^{+}$conformally onto $\Delta-((-1,0])$, and it maps both of $\pm i \tanh (-x)^{1 / 2}$ and $-\tanh ^{2}(-x)^{1 / 2}$. Thus the cut made by $\vartheta$ is repaired by the square function (by Schwarz's Reflection Principle): $\varphi_{i}$ is continuous at all points of $(-\infty, 0]$ and therefore analytic on $D_{i}$. Because $\varphi_{i}(z) \in(-1,0]$ if and only if $z \in(-\infty, 0]$ the injectivity of $\varphi_{i}$ on $D_{i}$ is clear.

Let $\gamma \subseteq D_{i}$ be a type III arc. Assume that $y>0$ and $x<0$ when $z=x+i y \in \gamma$. Let $\gamma^{\prime}=\vartheta(\gamma)$ so that $\gamma^{\prime} \subseteq S$. We show that $\gamma^{\prime}$ is as in Lemma 20 so that $\tan ^{2} \gamma^{\prime}$ has the restriction property in $\Delta$ and, hence, $\gamma$ has the restriction property in $D_{i}$.

Let $z=x+i y$ be an arbitrary point of $\gamma$ and write

$$
z^{1 / 2}=u+i v
$$

for the corresponding point $\vartheta(z) \in \gamma^{\prime}$; then

$$
x+i y=u^{2}-v^{2}+2 i u v .
$$

Eliminating $v$, and remembering that $x<0$, we see that

$$
\begin{aligned}
u^{2} & =\frac{1}{2}\left(x+\left(x^{2}+y^{2}\right)^{1 / 2}\right) \\
& =\frac{|x|}{2}\left(\left(1+\frac{y^{2}}{x^{2}}\right)^{1 / 2}-1\right) .
\end{aligned}
$$

Since $y^{2} / x^{2}<1$ (observation (vii)), the binomial series implies that

$$
\begin{aligned}
u^{2} & =\frac{y^{2}}{4|x|}-\frac{1}{16} \frac{y^{4}}{|x|^{3}}+\cdots \\
& \sim \frac{y^{2}}{4|x|},
\end{aligned}
$$


as $z$ tends to $\infty$ along $\gamma$. It follows from (34) that

$$
\lim _{t \rightarrow \infty} u^{2}=a \text { exists, } \quad 0 \leq a \leq\left(\frac{\pi}{4}\right)^{2} .
$$

Now let $h$ be the arc-length parametrization of $\gamma$ and write $h(t)=x(t)+i y(t)$. Let $k=\vartheta \circ h=h^{1 / 2}$ so that $k$ parametrizes $\gamma^{\prime}$. Write $k(t)=u(t)+i v(t)$. (i), (ii), (iii), and (iv) of Lemma 20 can now be verified.

Obviously $k(t) \in S$, for all $t \geq 0$, so (i) is true. As $t \rightarrow$ $\infty,|k(t)|=|h(t)|^{1 / 2} \rightarrow \infty$, but since $0 \leq u(t) \leq \pi / 4$ we must have $v(t) \rightarrow+\infty$, so that (ii) is true. Item (iii) follows from (46). Now $h(t) \rightarrow \infty$ as $t \rightarrow \infty,\left|h^{\prime}(t)\right| \equiv 1$, and $h^{\prime}(t) \rightarrow-1$ as $t \rightarrow \infty$. So, by Lemma 16 ,

$$
\lim _{t \rightarrow \infty} \frac{k^{\prime}(t)}{\left|k^{\prime}(t)\right|}=\frac{|h(t)|^{1 / 2}}{h(t)^{1 / 2}} \frac{h^{\prime}(t)}{\left|h^{\prime}(t)\right|} \longrightarrow-i(-1)=i .
$$

So (iv) is true and we have now completed the proof.

4.4. Case 4: $C_{i}$ Is a Hyperbola Component. We can deal simultaneously with the convex and concave complementary domains of a hyperbola component as follows. Let $-\pi / 2<$ $\alpha<\pi / 2$ and let $C_{i}=\sin (\alpha+i \mathbb{R})$. If $\alpha<0, C_{i}$ is the arc

$$
C_{i}=\left\{z=x+i y \in \mathbb{C}: x<0, \frac{x^{2}}{\sin ^{2} \alpha}-\frac{y^{2}}{\cos ^{2} \alpha}=1\right\},
$$

and if $\alpha>0, C_{i}$ is the arc

$$
C_{i}=\left\{z=x+i y \in \mathbb{C}: x>0, \frac{x^{2}}{\sin ^{2} \alpha}-\frac{y^{2}}{\cos ^{2} \alpha}=1\right\} .
$$

Let $D_{i}$ be the complementary domain to the "left" of $C_{i}$; then $D_{i}$ is convex when $\alpha<0$ and concave when $\alpha>0$. Linear equivalence will be used as before to reduce the general case to this one.

The function $\sin ^{-1}$ maps the double cut plane $\mathbb{C}$ $\{(-\infty,-1] \cup[1, \infty)\}$ conformally onto the vertical strip $|x|<$ $\pi / 2$, mapping the upper/lower parts of the first domain onto the upper/lower parts of the second. The upper and lower limits of $\sin ^{-1}$ at a point $-x \in(-\infty,-1]$ are $-\pi / 2 \pm i \cosh ^{-1} x$. The arc $C_{i}=\sin (\alpha+i \mathbb{R})$ is mapped to the line $\operatorname{Re} z=\alpha$. Therefore $\sin ^{-1}$ maps $D_{i}-(-\infty,-1]$ conformally onto the strip

$$
D_{\alpha}=\left\{z=x+i y \in \mathbb{C}:-\frac{\pi}{2}<x<\alpha\right\}
$$

If

$$
\lambda(z)=\frac{\pi}{4} \frac{z+(\pi / 2)}{\alpha+(\pi / 2)},
$$

then $\lambda$ maps $D_{\alpha}$ conformally onto the strip

$$
S=\left\{z=x+i y \in \mathbb{C}: 0<x<\frac{\pi}{4}\right\} .
$$

Therefore

$$
\varphi_{i}(z)=\tan ^{2} \lambda\left(\sin ^{-1} z\right)
$$

is a Riemann mapping function for $D_{i}$. Now let $\gamma$ be a type III arc in $D_{i}$. As in Case 3 the case $\gamma \subseteq \mathbb{R}$ is trivial, so we can assume that $\gamma$ lies entirely in the upper half-plane. It will be sufficient for us to show that $\lambda\left(\sin ^{-1} \gamma\right)$ has a parametric function $k$ as in Lemma 20.

Let $z=x+i y$ be arbitrary point of $\gamma$ and write $\sin ^{-1} z=$ $u+i v$ for the corresponding point of $\sin ^{-1} \gamma$. Clearly, by (50),

$$
u+i v \in D_{\alpha}
$$

Now

$$
z=x+i y=\sin (u+i v)=\sin u \cosh v+i \cos u \sinh v
$$

so that

$$
|z|^{2}=\sin ^{2} u \cosh ^{2} v+\cos ^{2} u \sinh ^{2} v=\sin ^{2} u+\sinh ^{2} v .
$$

As $z \rightarrow \infty$ along $\gamma,|z|^{2} \rightarrow+\infty$ and $\sin ^{2} u$ remains bounded; therefore

$$
v \longrightarrow+\infty \text { as } z \longrightarrow \infty \text { along } \gamma .
$$

It now follows from (56) and (57) that

$$
\sin u=\frac{x}{|z|}\left(\tanh ^{2} v+\frac{\sin ^{2} u}{\cosh ^{2} v}\right)^{1 / 2} \sim \frac{x}{|z|} \quad \text { as } z \longrightarrow \infty .
$$

Let $h$ be the arc-length parametrization of $\gamma$. As $z \rightarrow$ $\infty$ along $\gamma$ its unit tangent vector has a limit $e^{i \theta}$, say. The asymptotes of $C_{i}$ are the rays $\arg z= \pm(\pi / 2-\alpha)$. Therefore

$$
\lim _{t \rightarrow \infty} \frac{h^{\prime}(t)}{\left|h^{\prime}(t)\right|}=\lim _{t \rightarrow \infty} h^{\prime}(t)=e^{i \theta}, \quad \text { where } \frac{\pi}{2}-\alpha \leq \theta \leq \pi .
$$

So, by (57) and Lemma 16,

$$
\lim _{t \rightarrow \infty} \frac{h(t)}{|h(t)|}=e^{i \theta}
$$

Now $g=\sin ^{-1} \circ h$ is a parametric function for $\sin ^{-1} \gamma$. By (54) it follows that

(i) $g(t) \in D_{\alpha}(t \geq 0)$, and (57) shows that

(ii) $\operatorname{Im} g(t) \rightarrow+\infty$ as $t \rightarrow \infty$.

Equation (60) shows that

(iii) $\lim _{t \rightarrow \infty} \operatorname{Re} g(t)=\sin ^{-1} \cos \theta=(\pi / 2)-\theta$ and we notice that $-\pi / 2 \leq(\pi / 2)-\theta \leq \alpha$, by (59).

Finally observe that

$$
\frac{g^{\prime}(t)}{\left|g^{\prime}(t)\right|}=\frac{\left|1-h(t)^{2}\right|^{1 / 2}}{\left(1-h(t)^{2}\right)^{1 / 2}} \frac{h^{\prime}(t)}{\left|h^{\prime}(t)\right|} \text {. }
$$

Now in the upper half-plane $\left(1-w^{2}\right)^{1 / 2} \sim-i w$, as $w \rightarrow$ $\infty$. So, as $t \rightarrow \infty$,

$$
\frac{g^{\prime}(t)}{\left|g^{\prime}(t)\right|} \sim \frac{|h(t)|}{-i h(t)} \frac{h^{\prime}(t)}{\left|h^{\prime}(t)\right|},
$$


and therefore

(iv) $\lim _{t \rightarrow \infty}\left(g^{\prime}(t) /\left|g^{\prime}(t)\right|\right)=i$.

It follows easily that $k=\lambda \circ g$ satisfies the hypothesis of Lemma 20, and therefore $\varphi_{i}(\gamma)$ has the restriction property in $\Delta$.

\section{Conflict of Interests}

The author declares that there is no conflict of interests regarding the publication of this paper.

\section{References}

[1] P. L. Duren, Theory of $H^{p}$ Spaces, Pure and Applied Mathematics, Vol. 38, Academic Press, New York, NY, USA, 1970.

[2] P. L. Duren, "Smirnov domains," Journal of Mathematical Sciences, vol. 63, no. 2, pp. 167-170, 1993.

[3] G. M. Goluzin, Functions of a Complex Variable, American Mathematical Society, Providence, RI, USA, 1969.

[4] D. Khavinson, "Factorization theorems for different classes of analytic functions in multiply connected domains," Pacific Journal of Mathematics, vol. 108, no. 2, pp. 295-318, 1983.

[5] I. I. Privalov, Boundary Properties of Analytic Functions, GITTL, Moscow, Russia, 1950, (Russian; German translation: Deutscher, Berlin, Germany, 1956).

[6] A. M. Ruben and P. Rosenthal, An Introduction to Operators on the Hardy-Hilbert Space, Springer, New York, NY, USA, 2007.

[7] Y. Soykan, "On equivalent characterisation of elements of Hardy and Smirnov spaces," International Mathematical Forum, vol. 2, no. 24, pp. 1185-1191, 2007.

[8] Y. Soykan, “On the continuity of restriction maps," International Journal of Mathematical Analysis, vol. 2, no. 17-20, pp. 823-826, 2008.

[9] Y. Soykan, "Restriction maps," International Journal of Mathematical Analysis, vol. 5, no. 29-32, pp. 1491-1496, 2011.

[10] V. P. Havin, "Boundary properties of integrals of Cauchy type and of conjugate harmonic functions in regions with rectifiable boundary," Matematicheskii Sbornik, vol. 68, no. 110, pp. 499517, 1965.

[11] G. David, "Singular integral operators over certain curves in the complex plane," Annales Scientifiques de l'École Normale Supérieure. Quatrième Série, vol. 17, no. 1, pp. 157-189, 1984.

[12] Y. Soykan, "Boundary behaviour of analytic curves," International Journal of Mathematical Analysis, vol. 1, no. 1-4, pp. 133157, 2007.

[13] C. Cowen and B. MacCluer, Composition Operators on Spaces of Analytic Functions, vol. 20 of Studies in Advanced Mathematics, CRC Press, New York, NY, USA, 1995.

[14] Y. Soykan, "Restriction maps on the Hardy spaces of the unit disc," International Journal of Mathematical Analysis, vol. 7, no. 49-52, pp. 2407-2411, 2013. 


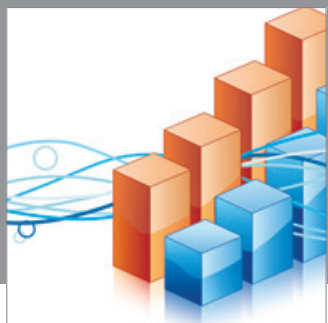

Advances in

Operations Research

mansans

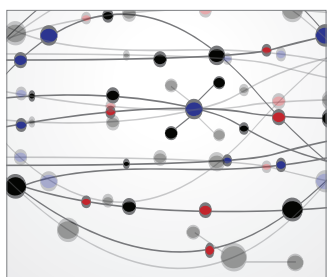

The Scientific World Journal
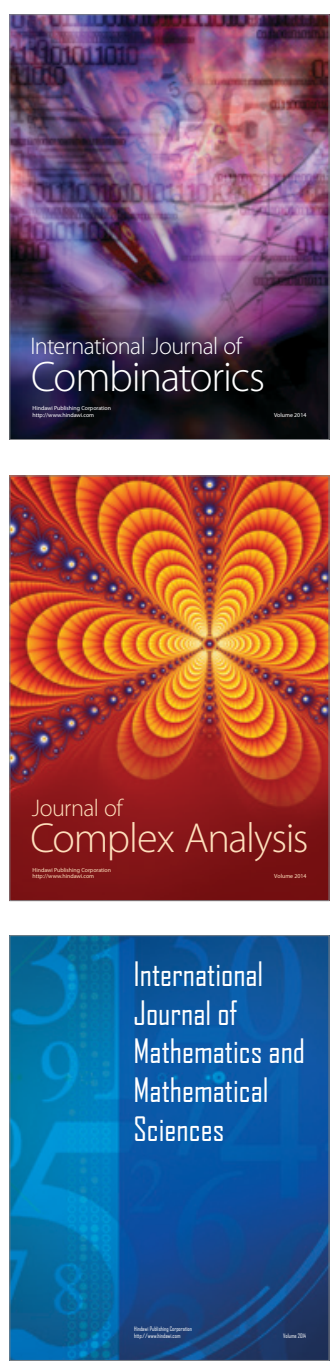
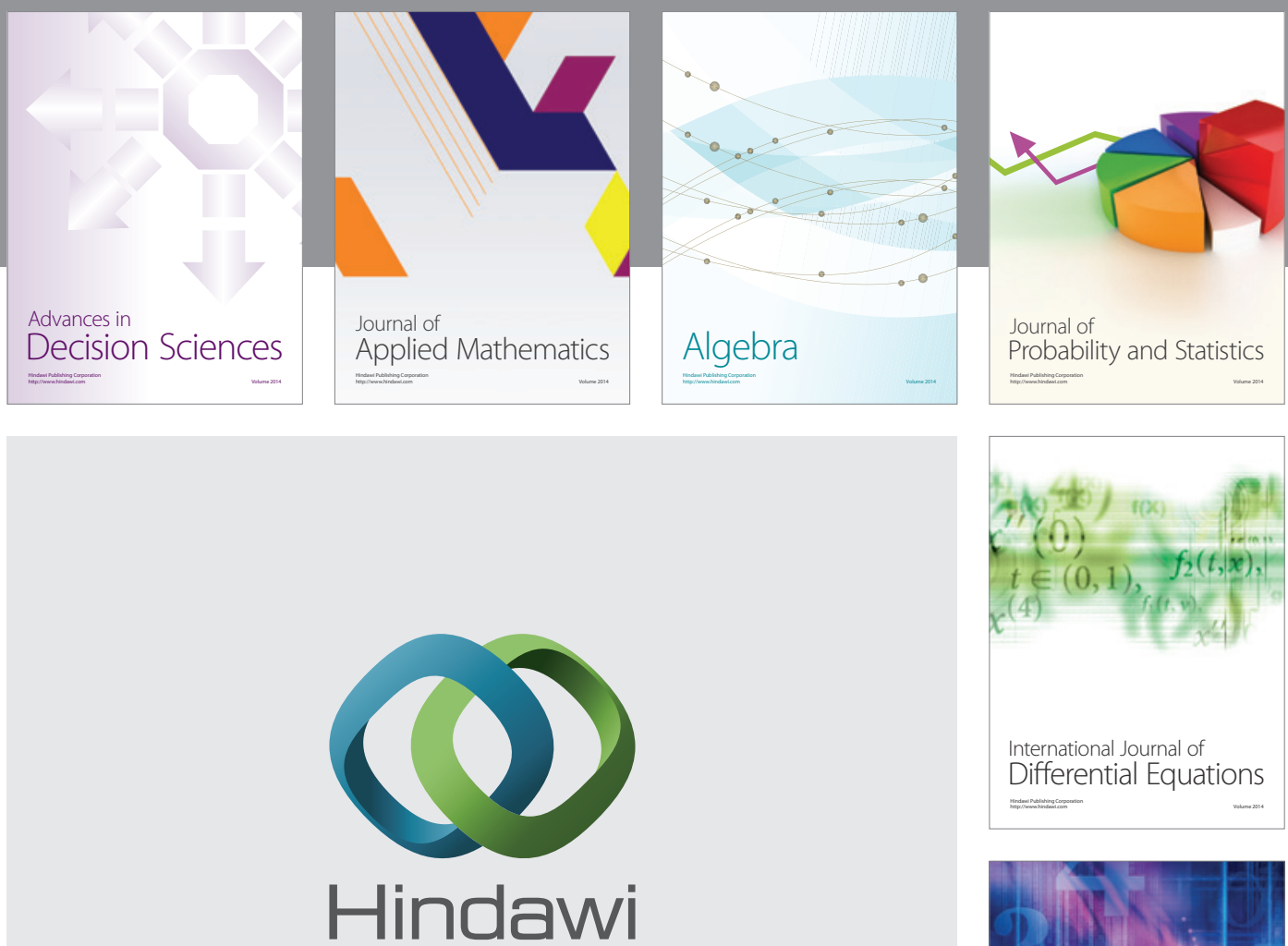

Submit your manuscripts at http://www.hindawi.com
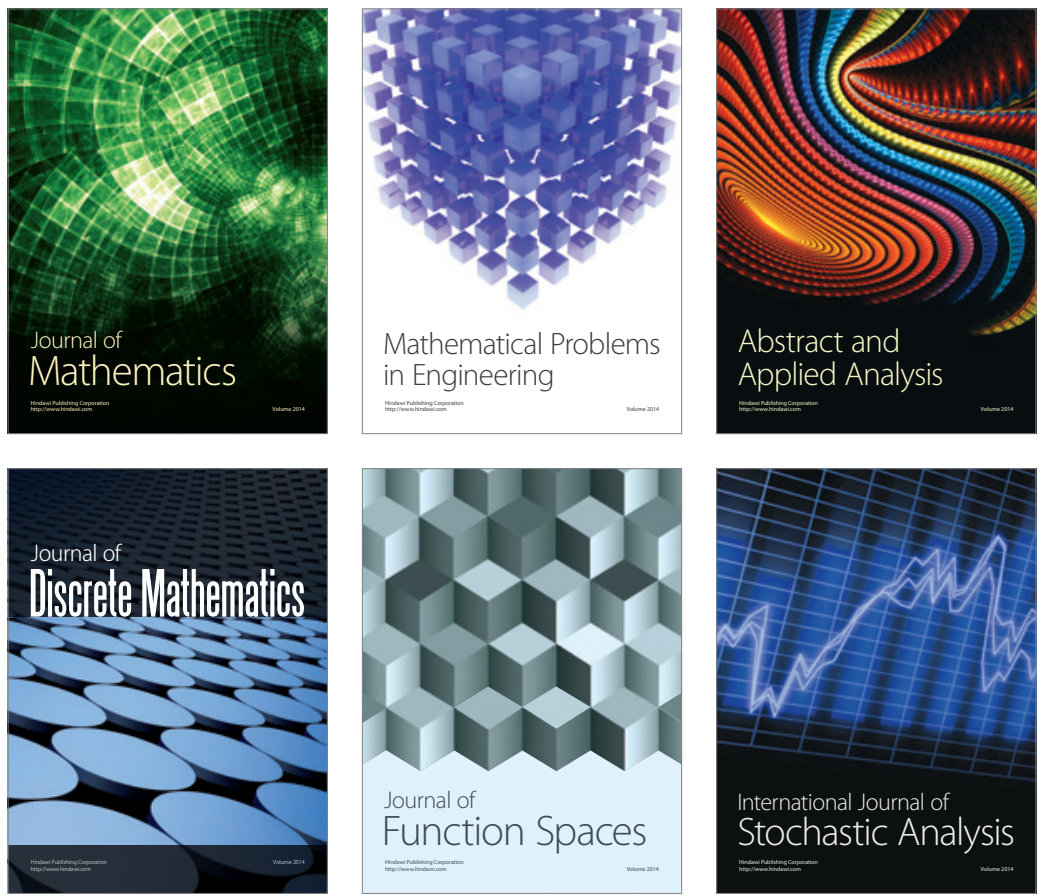

Journal of

Function Spaces

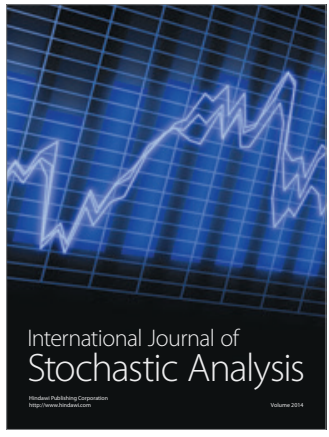

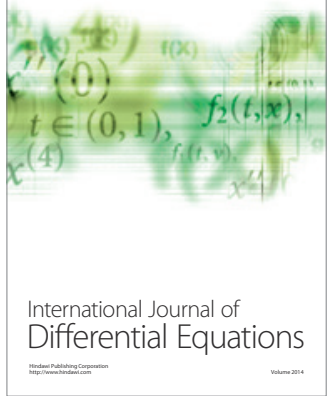
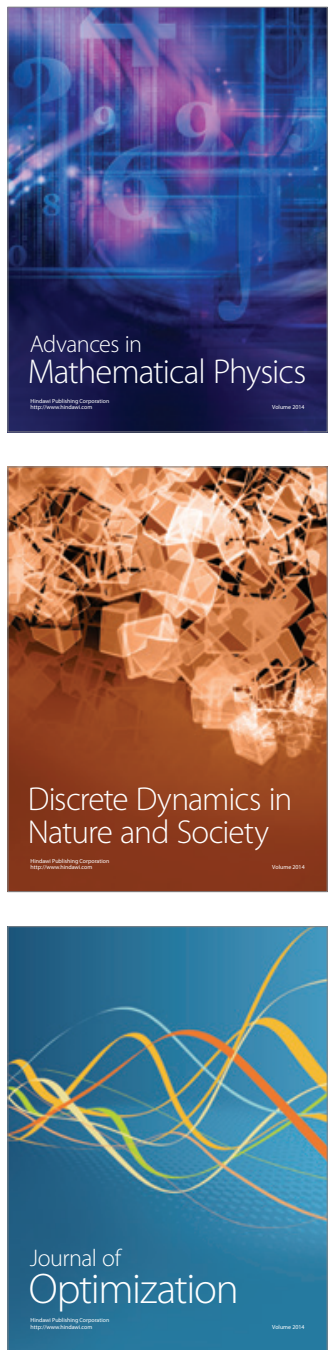